\title{
El fomento de los valores sociales en el aula y la constitución de una sociedad cívica, informada, crítica y seria a través del aprendizaje
}

\author{
The promotion of social values in the classroom and the \\ constitution of a civic, informed, critical and serious society \\ through learning
}

\author{
Mª Nieves Alonso García ${ }^{1}$
}

Fecha de recepción: 12/19/2018; Fecha de revisión: 25/02/2019; Fecha de aceptación: 30/04/2019

Cómo citar este artículo:

Alonso, M.N (2019). El fomento de los valores sociales en el aula y la constitución de una sociedad cívica, informada, crítica y seria a través del aprendizaje, Revista de Innovación y Buenas Prácticas Docentes, 8 (2), 116-122.

Autor de Correspondencia: marianieves.alonso@ui1.es

\begin{abstract}
Resumen:
En la presente contribución se aborda una propuesta de innovación docente centrada en contribuir a la mejora del proceso de enseñanza-aprendizaje sobre el fundamento de los artículos 27.2 y 54 de la Constitución Española favoreciendo los procesos educativos transversales. En los mencionados artículos se reconoce el derecho a la educación y su objeto, basado en el pleno desarrollo de la personalidad humana en el respeto a los principios democráticos de convivencia y a los derechos y libertades fundamentales y se configura la institución del Defensor del Pueblo como alto comisionado de las Cortes Generales, respectivamente.

El objetivo perseguido en esta propuesta es fomentar una educación en valores para desarrollarse en un entorno de respeto a los derechos fundamentales a través del aprendizaje de las instituciones del Estado. La enseñanza debe perseguir a través de la adquisición de conocimientos la formación de una sociedad cívica, informada, crítica y seria.
\end{abstract}

Palabras clave: innovación docente, valores sociales, enseñanza-aprendizaje

\begin{abstract}
:
This contribution deals with a proposal for teaching innovation focused on contributing to the improvement of the teaching-learning process on the basis of Articles 27.2 and 54 of the Spanish Constitution, favouring transversal educational processes. The articles recognise the right to education and its object, based on the full development of the human personality in respect for the democratic principles of coexistence and fundamental rights and freedoms, and the institution of the Ombudsman is configured as a high commissioner of the Cortes Generales, respectively.

The objective pursued in this proposal is to promote an education in values to develop in an environment of respect for fundamental rights through the learning of State institutions. The teaching must pursue through the acquisition of knowledge the formation of a civic, informed, critical and serious society.
\end{abstract}

Key Words: teaching innovation, social values, teaching-learning

\footnotetext{
${ }^{1}$ Universidad Isabel I, marianieves.alonso@ui1.es, https://orcid.org/0000-0002-1091-6819
} 


\section{INTRODUCCIÓN}

El artículo 27. 2 de la Constitución Española proclama que "la educación tendrá por objeto el pleno desarrollo de la personalidad humana en el respeto a los principios democráticos de convivencia y a los derechos y libertades fundamentales".

Esta reflexión planteada por nuestros constituyentes constituirá la esencia de la presente contribución en la cual se plantea una propuesta de innovación docente encaminada a dar a conocer a los alumnos la institución del Defensor del Pueblo a nivel estatal y sus figuras homólogas a nivel autonómico, así como la del Defensor del Pueblo Europeo en el ámbito de la Unión Europea.

Su objetivo principal, al margen de acercar dicha institución a los estudiantes, será formar a ciudadanos conscientes con la importancia de velar por la protección de sus derechos fundamentales, así como, por el respeto de los mismos en la esfera de sus relaciones sociales, fundamentalmente centradas en el aula como lugar en donde los alumnos construyen sus redes y vínculos.

El principal objetivo que se persiguen con esta propuesta de innovación se centra en contribuir a la mejora del proceso de enseñanza-aprendizaje sobre el fundamento de los artículos 27.2 y 54 de la Constitución Española favoreciendo los procesos educativos transversales. En los mencionados artículos se reconoce el derecho a la educación y su objeto, basado en el pleno desarrollo de la personalidad humana en el respeto a los principios democráticos de convivencia y a los derechos y libertades fundamentales y se configura la institución del Defensor del Pueblo como alto comisionado de las Cortes Generales, respectivamente.

Dicho objetivo general se desarrollará a partir de objetivos específicos: realizar un reto instructivo en el ámbito de la educación que vincule a los docentes del ámbito universitario con las principales instituciones del Estado a partir de un proceso multidisciplinar de cooperación; orientar en el proceso de realización de quejas al Defensor del Pueblo; favorecer la autonomía del alumno de cara a manifestar un problema en la esfera de la convivencia escolar; fortalecer la coordinación entre estudiantes e instituciones públicas; desarrollar sesiones prácticas vinculadas con los contenidos teóricos difundidos a lo largo de las sesiones magistrales y consolidar un pensamiento reflexivo en los alumnos sobre la necesidad de proteger y respetar los derechos fundamentales a fin de consolidad una sociedad cívica, informada, crítica y seria.

\section{DESARROLLO DE LA EXPERIENCIA DE INNOVACIÓN}

La presente propuesta de innovación estará constituida por un equipo multidisciplinar dentro del ámbito jurídico. El grupo de trabajo se formará por miembros pertenecientes a distintas áreas de conocimiento, íntimamente ligadas entre sí. De este modo, la propuesta engloba disciplinas directamente vinculadas con los principales objetivos de la misma. Por un lado, el Derecho Constitucional y por otro, el Derecho Administrativo. La primera de las ramas jurídicas tiene como objetivo velar por la existencia de una 
sociedad organizada sobre una base democrática y participativa, dentro de la cual adquiere especial relevancia la protección de los derechos fundamentales. La incorporación del Derecho Administrativo es significativa, ya que es la vertiente del Derecho público que se encarga de estudiar la organización y funciones de las instituciones del Estado. Desde este contexto, su contribución se centra en facilitar el conocimiento de la institución y en velar por la defensa de los derechos fundamentales que incida en la mejora de las relaciones personales entre los sujetos implicados.

La citada propuesta, se presentará enclavada, cómo se ha mencionado en líneas anteriores, en los contenidos curriculares de las asignaturas de Derecho Constitucional y Derecho Administrativo. La consolidación de conceptos en estas materias se revela complicada para los alumnos. Con el diseño y desarrollo de métodos alternativos más innovadores, basados en metodologías de aprendizaje-servicio (Puig, Martín \& Batlle, 2007) como el aquí descrito se pretende que los estudiantes se muestren más participativos, reflexivos y proactivos, lo que conllevará con una alta probabilidad a que los mismos amplíen la complejidad de procesos lógicos desde sus propios intereses y necesidades (Tejada, 2013). Es en esta línea en la que se propone el desarrollo de la propuesta.

La actividad se iniciará con la definición y exposición de los conceptos jurídicos básicos en torno a la figura del Defensor del Pueblo y finalizará con la ejecución práctica del mismo mediante la solución a los problemas detectados. En esta etapa se llevarán a cabo las siguientes actividades: construcción de una comunidad virtual de aprendizaje dónde el brainstorming y el intercambio de información útil se conviertan en técnicas de enseñanza entre los participantes. Esta comunidad virtual servirá también de punto de encuentro y comunicación siendo utilizada para la puesta en común de los avances relevantes; estudio de opciones y planificación de actividades para el diseño del caso práctico y búsqueda de información sobre el entorno y sus recursos para la construcción del caso práctico. En esta última etapa se llevará a cabo el diseño del caso práctico, el diagnóstico del problema, la búsqueda de soluciones y la elaboración de la propuesta final. Por último, los alumnos deberán presentación públicamente el caso práctico ante el resto de los compañeros y profesores de las disciplinas implicadas.

\subsection{Diseño y desarrollo de las sesiones teóricas de la propuesta de innovación.}

La presente propuesta se fundamenta en el paradigma socio-crítico referido a una metodología aprendizaje-servicio (Mendía, 2012) desde la que se plantea el diseño de una propuesta de innovación docente para promover en los estudiantes un acercamiento a la figura del Defensor del Pueblo.

En esta línea, se pretende desarrollar una propuesta de intervención pedagógica que incorpore aspectos teórico-prácticos relativos a la figura del Defensor del Pueblo contemplando una serie de actividades centradas en los ámbitos de investigación, análisis, reflexión y resolución de casos prácticos, mientras se promueven a su vez acciones educativas transversales que proporcionarán a los estudiantes una visión más completa y positiva de sí mismos, de los demás y de su entorno (Aranda et al., 2007).

En las sesiones teóricas se enseñará a los alumnos la regulación y funciones de la institución del Defensor del Pueblo. Entre otros contenidos, se enseñará a los alumnos acerca de esta figura, que se encuentra constitucionalmente reconocida en el artículo 54 y fue creada por la Ley 2/1994, de 9 de marzo, cuyo origen y fundamento hallamos en la LO 3/1981, de 6 de abril. El artículo 12 de la citada legitima la existencia de órganos similares en las Comunidades Autónomas (Álvarez, 2018).

El objetivo de estas sesiones teóricas consiste en que el alumno aprenda que la principal función del Defensor del Pueblo es velar por que se garantice a los ciudadanos 
el derecho a una buena administración y cuidar especialmente de proteger y defender los derechos de los menores y de todas las personas en peligro de exclusión social.

En cuanto a la defensa de los derechos comprendidos en el Título I de nuestra vigente Carta Magna, tal y como establece el artículo 54 CE y el artículo 1 de su Ley Orgánica, se articula por medio del control de la Administración. La mayor parte de las actuaciones del Defensor del Pueblo se orientan a asegurar esta fiscalización de la Administración en aras a cumplir los principios que de ella se predican: eficacia, jerarquía, coordinación y sometimiento pleno a la ley y al derecho.

El Defensor del Pueblo es un Alto Comisionado de las Cortes Generales y, en consecuencia, debe elevar a éstas un informe anual. A su vez, entre sus funciones de salvaguardia de la actividad de la Administración, se encuentran las propias recomendaciones que le puede efectuar. Estos informes y recomendaciones no han sido sólo instrumentos básicos sobre los que se ha cimentado el prestigio de esta institución, han constituido ocasionalmente impulsos de mejora legislativa o de actuación de la Administración.

Asimismo, será relevante que los alumnos conozcan la existencia de figuras homólogas a nivel autonómico, consecuencia de la descentralización política interna de administraciones autonómicas que ha originado la creación de defensores del pueblo específicos para cada una de las Comunidades Autónomas; así como la existencia del Defensor del Pueblo Europeo, fruto del proceso del proceso de integración europea.

En cuanto a las funciones y naturaleza del Defensor del Pueblo Europeo, será relevante que los alumnos sean conscientes de que el Defensor del Pueblo Europeo aparece en el Derecho de la Unión Europea como una persona física, elegida y nombrada por el Parlamento Europeo, para que reciba e instruya las reclamaciones de los ciudadanos europeos relativas a casos de mala administración en la actuación de las instituciones, organismos y órganos de la Unión, e informe de sus investigaciones y resultados tanto al ciudadano, como al órgano concernido y al Parlamento Europeo.

Se trata de una autoridad de disuasión que, a través de sus tres medios esenciales de actuación, como son la investigación, la recomendación y la publicidad de sus críticas, trata de influir y de persuadir, ayudar y orientar, y no de imponer, vencer u obligar. No es un juez, ni un tribunal, ni puede dar órdenes, ni modificar o anular actos administrativos. Ahora bien, el carácter no vinculante de sus decisiones no obsta para que obtenga el respeto de los ciudadanos si finalmente resulta eficaz en sus actuaciones, especialmente, en las que supongan la denuncia de actos que lesionen derechos de los ciudadanos o de búsqueda de soluciones a problemas particulares.

Está legitimado para presentar una reclamación al Defensor del Pueblo Europeo todo ciudadano de la UE o toda persona física o jurídica -empresa, asociación, fundación...- que resida o tenga su sede social en un Estado miembro de la Unión. Lo más efectivo es presentar la reclamación través de la página web del Defensor del Pueblo $^{2}$. Este derecho de reclamación se ha consolidado en la práctica institucional como una vía alternativa a la del recurso ante el juez comunitario para la defensa de los intereses por parte de los ciudadanos; una vía alternativa, es decir, extrajudicial, que responde a criterios específicos y no persigue los mismos que la vía judicial. El procedimiento de presentación es muy flexible y, además, gratuito. La reclamación

\footnotetext{
${ }^{2}$ http://www.ombudsman.europa.eu.
} 
puede formularse por escrito, en cualquiera de las lenguas reconocidas por el Tratado de la Unión Europea, utilizando un formulario que facilita la Secretaría del Defensor del Pueblo, pudiendo hacerse llegar por correo (postal o electrónico) o por fax. El Estatuto del Defensor del Pueblo exige que se presente en un plazo de dos años desde que se tuvo conocimiento de los hechos, pero la aplicación estricta de este requisito es discutible e incluso inoperante. La reclamación debe incluir con claridad el acto objeto de la misma, y la identidad del reclamante, aunque el Defensor del Pueblo podrá clasificarla como confidencial si lo considera necesario.

Uno de los aspectos más importantes de la misión del Defensor del Pueblo en todos sus niveles consiste en generar confianza a través del diálogo entre con ciudadanos. Parte de la función de un defensor del pueblo en cualquier democracia moderna es ocuparse de que la administración pública se oriente al servicio a los ciudadanos e intente satisfacer sus cada vez más elevadas expectativas.

En definitiva, los alumnos deben ser partícipes del entorno que les rodea estando al corriente de que cuando un ciudadano presente cierto descontento con una institución o se atente a sus derechos constitucionalmente garantizados, se le debe brindar la oportunidad de corregir tal situación. Y es el Defensor del Pueblo Europeo la institución donde se debe inferir la reclamación para que tenga conocimiento del problema.

\subsection{Diseño y desarrollo de las sesiones prácticas de la propuesta de innovación}

Las sesiones prácticas se desarrollarán en las últimas sesiones de la propuesta de innovación. Para ello, el docente expondrá a sus alumnos un marco contextual común en el ámbito escolar, al hilo del mismo y a partir del cual se irán conformando las distintas actividades prácticas en función de los avances perpetrados en el aula.

Es fundamental que el docente centre al alumno en el estado actual de la cuestión destacando que, en los últimos años, han aumentado los conflictos de convivencia escolar, tanto vinculados a agresiones como vinculados a los problemas de integración de los alumnos de origen extranjero.

Es importante poner en conocimiento de los alumnos que las quejas dirigidas al Defensor del Pueblo devienen en resultados. A modo de ejemplo, una queja suscitada por las agresiones sufridas por un niño de ocho años en el colegio, y la posterior remisión por parte del Procurador del Común de la Comunidad Autónoma de Castilla y León de una resolución a la Consejería de Educación, supuso la aprobación de la Orden EDU/1071/2017, de 1 de diciembre, por la que se establece el "Protocolo específico de actuación en supuestos de posible acoso en centros docentes, sostenidos con fondos públicos que impartan enseñanzas no universitarias de la Comunidad de Castilla y León"3 Asimismo, también se ha publicado la Orden EDU/1070/2017, de 1 de diciembre, por la que se establece el "Protocolo de actuación en agresiones al personal docente y no docente de los centros sostenidos con fondos públicos que imparten enseñanzas no universitarias de la Comunidad de Castilla y León"4.

En relación con la problemática generada por la integración de alumnos extranjeros, se recomendó que esta conflictividad se resolviera fundamentalmente, a través de la mediación como estrategia de resolución de conflictos (Pérez-Ugena, 2014), recordando a las familias que es preciso su deber de colaborar con el Centro para que el proceso educativo de los alumnos se lleve de forma adecuada, y, si fuera necesario,

\footnotetext{
${ }^{3} \mathrm{http} / / /$ www.educa.jcyl.es/es/resumenbocyl/orden-edu-1071-2017-1-diciembre-establece-protocoloespecif).

${ }^{4}$ http://www.educa.jcyl.es/es/resumenbocyl/orden-edu-1070-2017-1-diciembre-establece-protocoloactuaci).
} 
promoviendo la coordinación institucional para que las instituciones de ámbito sanitario, social o de otro tipo adopten medidas dirigidas a modificar aquellas circunstancias personales, familiares o sociales de los alumnos que puedan ser determinantes de la aparición y persistencia de conductas perturbadoras para la convivencia del centro.

El desarrollo de esta experiencia práctica podrá extrapolarse a los problemas de convivencia escolar generados en las aulas en todas los niveles educativos, en donde se fomentará la resolución consensuada de los conflictos.

La adquisición de conocimientos debe devenir en la formación de una sociedad cívica, informada, crítica y seria que se sustente en el pleno respeto de los derechos fundamentales.

\section{RESULTADOS}

Con la propuesta de innovación descrita a lo largo de estas líneas se pretende mejorar la calidad del proceso educativo usando el material interactivo generado, así como la consolidación de un grupo de investigación mixto constituido por disciplinas afines, que en su actuación conjunta fomenten el mejor aprendizaje por parte del alumno.

Asimismo, el trabajo colaborativo de los alumnos supondrá la administración de grupos de clase a través de la propuesta educativa y la construcción de entornos personales de aprendizaje. Fruto de esta experiencia, se potenciará el trabajo cooperativo y se impulsará la ruptura del distanciamiento entre los ciudadanos y las instituciones del Estado.

La actividad permitirá al alumno desarrollar las competencias generales de las asignaturas Derecho Constitucional y Derecho Administrativo a través del conocimiento de una parte de los contenidos curriculares de las materias mencionadas (Cano, 2015).

Los alumnos se implicarán en la búsqueda, obtención, procesamiento y comunicación de información (oral, impresa, audiovisual, digital o multimedia) para transformarla en conocimiento y aplicarla en los procesos de enseñanza y aprendizaje en las materias

En esta misma línea, se adquirirán estrategias para estimular el esfuerzo del estudiante y promover su capacidad para aprender por sí mismo y con otros, y desarrollar habilidades de pensamiento y de decisión que faciliten la autonomía, la confianza e iniciativa personales.

Como reto final de esta propuesta se pretenderá fomentar el espíritu crítico, reflexivo y emprendedor del alumno y el desarrollo de habilidades de aprendizaje que les permitan continuar estudiando de un modo que habrá de ser en gran medida autodirigido y autónomo.

\section{CONCLUSIONES}

La Declaración final de la Conferencia Mundial de Educación Superior organizada por la Unesco en 1998 estableció que "La educación superior ha de reforzar sus funciones de servicio a la sociedad y, más concretamente, sus actividades orientadas a erradicar 
la pobreza, la intolerancia, la violencia, el analfabetismo, el hambre, el deterioro del medio ambiente y las enfermedades, sobre todo con un planteamiento interdisciplinario y transdisciplinario para analizar los problemas y las dudas planteados".

Tomando como punto de partida esta reflexión, el objetivo perseguido en esta propuesta es fomentar una educación en valores para desarrollarse en un entorno de respeto a los derechos fundamentales a través del aprendizaje de las instituciones del Estado. La enseñanza debe perseguir a través de la adquisición de conocimientos la formación de una sociedad cívica, informada, crítica y seria.

El aula representa la esfera en la que los alumnos desarrollan sus relaciones sociales, constituyendo un espacio de construcción de redes y vínculos. Los trabajos colaborativos conducen a una dinámica en la que se engloban ideales, intereses, motivaciones y necesidades que rigen la conducta y las decisiones.

Es fundamental que los alumnos aprendan a analizar, programar, evaluar y trabajar en equipo con el resto del grupo, negociar y distribuir tareas, adquirir compromisos y tomar decisiones.

A través de la experiencia teórica de esta propuesta, el alumno se acercará a una institución fundamental de nuestro ordenamiento jurídico, profundizando en su conocimiento a su posterior puesta en práctica. El desarrollo práctico fomentará la convivencia pacífica en el aula y la resolución de conflictos a través de la mediación y las buenas prácticas.

\section{REFERENCIAS}

Álvarez, $\mathrm{M}^{\mathrm{a}} \mathrm{I}$. El Defensor del Pueblo y los defensores autonómicos: instituciones de garantía de los derechos. En La Constitución Española: 1978-2018, Madrid: Francis Lefebvre, 2018, pp. 1500-1599.

Aranda, R., Cerrillo, R., De la Herrán, A., De Miguel, S., Gómez, M.; Hernández-Castilla, R., Izuzquiza, D., Murillo, F.J., Pérez, M., Rodríguez-Izquierdo, R.Mª. \& Egido, I. (2007). El aprendizaje basado en problemas como innovación docente en la universidad. Educación y futuro: revista de investigación aplicada y experiencias educativas, posibilidades y limitaciones, 16, 85-100.

Cano, E. (2015). Evaluación por competencias en educación superior. Madrid: La Muralla.

Mendia, R. (2012). El Aprendizaje-Servicio como una estrategia inclusiva para superar las barreras al aprendizaje y a la participación. Revista Educación Inclusiva, 5 (1), 71-82.

Pérez-Ugena, M. (2014). Garantía del derecho a la tutela judicial efectiva en los sistemas principales de resolución de conflictos alternativos: arbitraje y mediación. Estudios de Deusto: revista de la Universidad de Deusto, 62 (1), 159-189.

Puig, J.M., Martín, X. \& Batlle, R. (2007). Cómo iniciar un proyecto de aprendizaje y servicio solidario. Bilbao: Zerbikas Fundazioa.

Tejada, J. (2013). La formación de las competencias profesionales a través del aprendizaje servicio. Cultura y Educación: Culture and Education, 25 (3), 285294. 